\title{
Infected deep vein thrombophlebitis in people who inject drugs: missed opportunities and potential for alternative antimicrobial approaches
}

\author{
Hugh McCaughan ${ }^{1,2} \cdot$ Clark D. Russell $^{1,3}$ [D $\cdot$ Dáire T. O'Shea ${ }^{1}$
}

Received: 18 August 2021 / Accepted: 22 October 2021 / Published online: 2 November 2021

(c) The Author(s) 2021

\begin{abstract}
Infected deep vein thrombophlebitis (i-DVT) in people who inject drugs (PWID) is a clinically challenging but poorly characterised disease. We undertook a retrospective observational study of 70 PWID presenting acutely with i-DVT to improve the clinical and microbiological characterisation of this disease. i-DVT was frequently associated with bacteraemia (59.1\% patients with blood cultures obtained), groin abscesses (in 34.3\%; of which 54.2\% required surgical drainage), and septic pulmonary emboli (38.6\%) requiring anticoagulation. Network analysis identified a cluster of patients presenting with respiratory symptoms but lacking typical DVT symptoms, more likely to have septic pulmonary emboli. A microbiologic diagnosis was frequently achieved (70\%). Causative pathogens were predominantly gram-positive (S. aureus and streptococci, especially anginosus group), whereas gram-negative pathogens were identified very infrequently (in $6.1 \%$ of microbiological diagnoses). This suggests routine empiric therapy against gram-negative bacteria, though commonly administered, is not required. High rates of clinical cure (88.6\%) were observed despite the complex nature of infections and independently of the highly variable intravenous and total antimicrobial durations received. There exists a rationale to devise pragmatic approaches to implement novel individualised treatment plans utilising oral antimicrobial therapy for i-DVT. Despite frequent healthcare interactions, opportunities to address HCV treatment and opioid substitution therapy were frequently missed during these acute admissions.
\end{abstract}

Keywords Opioid use disorder · Substance use disorders · Deep vein thrombosis · Thrombophlebitis

\section{Introduction}

People who inject drugs (PWID) are at increased risk of bacterial disease complicating injections, including cellulitis, abscesses and infective endocarditis (IE). Infected

Hugh McCaughan and Clark D. Russell contributed equally to this manuscript

Hugh McCaughan

hugh.mccaughan@ed.ac.uk

$\triangle$ Clark D. Russell

clark.russell@ed.ac.uk

1 Clinical Infection Research Group, Regional Infectious Diseases Unit, Western General Hospital, Edinburgh, UK

2 Centre for Synthetic and Systems Biology, Waddington Building, Kings Buildings, Edinburgh, UK

3 University of Edinburgh Centre for Inflammation Research, Queen's Medical Research Institute, Edinburgh, UK deep vein thrombophlebitis (i-DVT) is estimated to account for up to $11 \%$ of infectious complications amongst hospitalised PWID, based on a single-centre Swiss retrospective observational study [1]. I-DVT is a clinically challenging entity but poorly characterised. Optimal management is not defined, particularly in comparison to IE in PWID [1-3]. In general, PWID are a therapeutically disenfranchised group, often excluded from potentially relevant clinical trials, contributing to a lack of data to guide therapeutic decisions. To improve the clinical characterisation of this entity, we undertook a retrospective observational study of PWID presenting acutely with i-DVT. 


\section{Methods}

\section{Case acquisition}

Cases were defined as (1) adults currently injecting drugs intravenously, presenting with (2) radiologically confirmed upper or lower limb DVT (excluding isolated superficial thrombophlebitis) with (3) evidence of infection, either (i) radiological or intra-operative (inflammatory vessel changes associated with thrombus, gas in thrombus, vessel with thrombus contiguous with abscess) or (ii) microbiological (bacteraemia or culture-positive intra-operative thrombus sample). Electronic patient records and Infectious Disease consultation records were searched for the terms "DVT", "PWID" and "IVDU" for patients admitted to infectious disease, vascular surgery or critical care wards from January 2017-December 2018 inclusive, identifying 2957 acute admissions, 70 of which met the case definition and had available records. This work was approved by the NHS Lothian Infection Service Quality Improvement Team.

\section{Analysis}

Microbiological cure was defined as a negative blood culture (BC) following a positive BC. Clinical cure was defined as no re-admission due to the same infection within one year of discharge. Symptom clusters were identified by network analysis using the Markov Clustering Algorithm (Graphia, version 2.0 [4]). Groups were compared using unpaired t-tests, Mann-Whitney tests or Fisher's exact tests as appropriate.

\section{Results}

\section{Cohort characteristics}

Patients were predominantly male $(46 / 70 ; 65.7 \%)$ with a median age of 37 years (Table 1, Table S1). Prior to admission 10/70 (14.3\%) patients had no fixed abode and 10/70 (14.3\%) resided in non-permanent accommodation. All patients injected heroin with 41/70 (58.6\%) using additional substances, most frequently cocaine and/ or benzodiazepines (Table 1; Figure S1). Active hepatitis $\mathrm{C}$ was present in $22 / 70$ (31.4\%), with 7 of these being new diagnoses during the index hospitalisation. Thirtysix patients $(51.4 \%)$ had previously been hospitalised due to complications of injection drug use (median of 1 , IQR $1-3$, range $1-15$ ), commencing a median of 33 months
Table 1 Cohort characteristics

\begin{tabular}{|c|c|}
\hline Variable & $\begin{array}{l}N(\%) \\
(n=70)^{\mathrm{a}}\end{array}$ \\
\hline \multicolumn{2}{|l|}{ Demographics } \\
\hline Male:Female & 46:24 \\
\hline Age, median (IQR) years & $37(32-41)$ \\
\hline Temporary accommodation or no fixed abode & $20(28.6)$ \\
\hline \multicolumn{2}{|l|}{ Substance use } \\
\hline Alcohol excess & $10(14.3)$ \\
\hline Heroin only & $29(41.4)$ \\
\hline + Cocaine & $26(37.1)$ \\
\hline + Benzodiazepines & $26(37.1)$ \\
\hline +Cannabis & $9(12.9)$ \\
\hline+ New psychoactive substance & $5(7.1)$ \\
\hline + Others & $6(8.6)$ \\
\hline \multicolumn{2}{|l|}{ Blood borne viruses } \\
\hline HIV positive & 0 \\
\hline Active HBV & $2(2.9)$ \\
\hline Active $\mathrm{HCV}$ & $22(31.4)$ \\
\hline Prior cleared HCV & $15(21.4)$ \\
\hline \multicolumn{2}{|l|}{ Presentation } \\
\hline Symptom duration prior to admission, median (IQR) days & $4.5(3-7)$ \\
\hline \multicolumn{2}{|l|}{ Vital signs, median (IQR) } \\
\hline Pulse, beats/min & $102(85-118)$ \\
\hline Systolic BP, mmHg & $113(90-123)$ \\
\hline $\mathrm{SpO}_{2}, \%$ & $97(95-99)$ \\
\hline Temperature, ${ }^{\circ} \mathrm{C}$ & $38.4(37.5-39)$ \\
\hline Respiratory rate, breaths/min & $17(16-19)$ \\
\hline \multicolumn{2}{|l|}{$\begin{array}{l}\text { Antimicrobial durations, } \\
\text { median (IQR) days }{ }^{\mathrm{b}}\end{array}$} \\
\hline Intravenous & $18(7-29)$ \\
\hline Oral follow-on $(n=54)$ & $14(14-27)$ \\
\hline Combined & $29(28-43)$ \\
\hline \multicolumn{2}{|l|}{ Surgical intervention } \\
\hline Abscess drainage & $13(18.6)$ \\
\hline Pseudoaneurysm ligation & $4(5.7)$ \\
\hline \multicolumn{2}{|l|}{ Complications } \\
\hline Bacteraemia & $39 / 66(59.1)^{c}$ \\
\hline Septic pulmonary emboli & 27 (38.6) \\
\hline Groin abscess & $24(34.3)$ \\
\hline Arterial involvement & $8(11.4)$ \\
\hline Pseudoaneurysm & $6(8.6)$ \\
\hline Splenic emboli & $3(4.3)$ \\
\hline Infective endocarditis & $3(4.3)$ \\
\hline \multicolumn{2}{|l|}{ Outcomes } \\
\hline Clinical cure & $62(88.6)$ \\
\hline Microbiological cure & $38 / 39(97.4)$ \\
\hline Substance-use related admission in year after discharge & $32(45.7)$ \\
\hline
\end{tabular}

Data shown as $n(\%)$ unless otherwise stated

${ }^{a}$ Denominator $=70$ unless otherwise stated

${ }^{\mathrm{b}}$ Data available for $n=68$

${ }^{\mathrm{c}} \mathrm{Blood}$ cultures obtained in $n=66$

$I Q R$ interquartile range, $H I V$ human immunodeficiency virus, $H B V$ hepatitis $\mathrm{B}$ virus, $\mathrm{HCV}$ hepatitis $\mathrm{C}$ virus, $\mathrm{BP}$ blood pressure, $\mathrm{SpO}_{2}$ oxygen saturations 
(IQR 20-64.75) previously, commonly due to cellulitis $(n=28)$ and/or deep vein thrombosis/pulmonary embolism (DVT/PE; $n=19)$.

\section{Presenting features}

Network analysis identified distinct symptom clusters. Combinations of leg pain with/without swelling and fever were the most common presenting symptoms, but one cluster of patients presented predominantly with fever and respiratory symptoms ( $\geq 1$ of cough, dyspnoea or pleuritic chest pain; Table S1, Figure S2). Laboratory evidence of systemic inflammation was common (median CRP $220 \mathrm{mg} / \mathrm{L}$ [IQR 175-292]; median neutrophils $9.8 \times 10^{9} / \mathrm{L}$ [6.9-13.9]) but overt sepsis (qSOFA $\geq 2$ ) was uncommon $(7 / 60 ; 11.7 \%$ ) (Table 1, Figure S3). Thirteen patients were admitted to the intensive care unit. Radiologic confirmation of DVT was by US (46/49; 93.9\%) and/or CT (36/55; 65.6\%), identifying thrombi most commonly in the common femoral $(n=29)$, external iliac $(n=17)$ or common iliac $(n=14)$ veins. Abnormalities were present in 21/61 (34.4\%) chest x-rays (cavitating pneumonia in four). Thoracic CT (pulmonary angiogram 24; venous contrast 13) identified definite emboli in $6 / 37$ $(16.2 \%)$ and cavitation in $23 / 37(62.2 \%)$ patients who underwent imaging; more often in the respiratory symptom cluster.

\section{Microbiological findings}

Bacteraemia was identified in 39/66 (59.1\%) patients with $\mathrm{BC}$ obtained at presentation (9 polymicrobial). Bacteraemic patients had a median of 1 positive $\mathrm{BC}$ per organism (range 1-3). Seven patients had $>1$ positive BC; in all cases Staphylococcus aureus. Intra-operative samples were culture-positive from 16/19 (84.2\%) patients where obtained. Considering $\mathrm{BC}$ and intra-operative samples together, a microbiological diagnosis was made in $49 / 70$ (70\%) patients; monomicrobial in 32/49 (65.3\%). Most monomicrobial infections were due to $S$. aureus $(21 / 32,65.6 \%)$. $S$. aureus, streptococci (especially anginosus group) and anaerobes were the most commonly identified pathogens overall (Table S2). Gram-negative organisms were infrequently identified (3 patients).

\section{Antimicrobial therapy}

All patients ( $n=68$ data available) were initially treated with intravenous antimicrobials continuing for a median of 18 days (IQR 7-29), with oral follow-on therapy in 54/68 (79.4\%) patients, prescribed for a median of 14 days (IQR 14-27), resulting in a median total duration of 29 days (IQR 28-43) but with substantial variation (Fig. 1A). The median inpatient stay was 24 days (IQR 12-29). Adherence to oral antimicrobial therapy after discharge is unknown, thus these data are the upper bound to oral and total durations. An enforced early IV-to-oral switch occurred in three patients due to intravenous access difficulties and in eight due to discharge against medical advice.

Initial empiric antimicrobial therapy favoured $S$. aureus coverage, with $65 / 69(94.2 \%)$ patients receiving agents with activity against gram-positive pathogens including $S$. aureus (Fig. 1C; Table S3). In 40/69 (58\%) patients, initial empiric therapy also included gram-negative and anaerobic coverage. Total antimicrobial days were summed and the proportion accounted for by each 'spectrum' was derived (Table S4). Specific anti-staphylococcal gram-positive therapy accounted for $42.4 \%$ of antimicrobial days, followed by anaerobic therapy (24.9\%), broad-spectrum therapy (13.0\%), narrow-spectrum gram-positive therapy $(9.9 \%)$ and gramnegative therapy $(8.8 \%)$.

\section{Other management}

Peripherally inserted central catheters or midlines, inserted by an anaesthetist or radiologist (excluding central venous access for vasopressors), were required for intravenous access in 32/70 (45.7\%) patients. Surgical intervention was required in 22/70 (31.4\%) patients, most commonly for abscess drainage $(n=13)$ or pseudoaneurysm ligation $(n=4)$. Therapeutic anticoagulation was initiated with subcutaneous low molecular weight heparin (LMWH) for most patients (57/70; 81.4\%) or LMWH followed by a direct-acting oral anticoagulant (DOAC; $\mathrm{n}=7)$ or DOAC alone $(n=1)$. Four patients were not anticoagulated and one was already taking therapeutic anticoagulation. The majority of patients received long-term opioid substitution therapy, however, 14/70 (20\%) patients were discharged without a treatment plan (Fig. 1D). Sixteen (of 22; 72.7\%) patients with active HCV infection were offered outpatient treatment; only six ultimately received treatment. In five patients active $\mathrm{HCV}$ infection was not addressed. Data was unavailable for one patient with active HCV.

\section{Complications}

Septic pulmonary emboli ( $n=27$ [38.6\%]; either definite emboli or cavitation) and groin abscesses ( $n=24$, [34.3\%]; of which 13 underwent drainage [54.2\%]) were the most common complications. Adjacent arterial involvement $(n=8 ; 11.4 \%)$ and pseudoaneurysms $(n=6 ; 8.6 \%)$ were less common. Other metastatic complications included: splenic emboli $(n=3 ; 4.3 \%)$, IE $(n=3$ [4.3\%]; 50/66 underwent echocardiography), vertebral osteomyelitis $(n=1 ; 1.4 \%)$, septic arthritis (metatarsophalangeal joint, $n=1 ; 1.4 \%$ ), pulmonary artery pseudoaneurysm requiring surgical intervention $(n=1 ; 1.4 \%)$, right ventricle thrombus containing an embolised needle tip $(n=1 ; 1.4 \%)$ and necrotising fasciitis of the groin and abdominal wall $(n=1 ; 1.4 \%)$. 
A

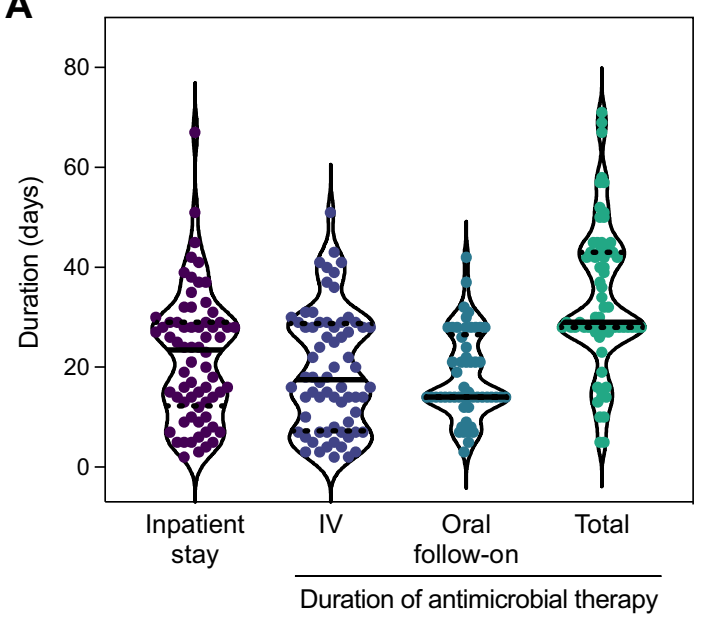

C

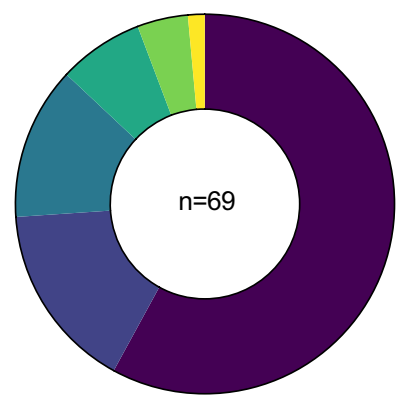

Initial empiric antimicrobial spectrum

- Gram positive (inc. S. aureus) + gram negative + anaerobic, $58.0 \%$

Gram positive (inc. S. aureus), $15.9 \%$

Gram positive (inc. S. aureus) + anaerobic, $13.0 \%$

Gram positive (inc. S. aureus) + gram negative, $7.2 \%$

Gram positive (exc. S. aureus) + gram negative + anaerobic, $4.3 \%$

Gram positive (exc. S. aureus), 1.4\%

Fig. 1 Inpatient management. A Duration of inpatient stay and antimicrobial therapy (intravenous [IV], oral, and total [IV and oral combined]). B Comparison of the duration of intravenous, inpatient and total antimicrobial therapy between patients with and without clinical cure. Data available for 68 patients. Groups compared by Mann Whit-

\section{Outcomes}

Amongst patients with data available, microbiological cure was achieved in 38/39 (97.4\%) cases. Clinical cure was achieved in $62 / 70(88.6 \%)$ cases and there were no deaths during the index admission. Clinical failure leading to re-admission occurred an average of 17 days after discharge ( $\mathrm{SD} \pm 9$, range 5-26). $32(45.7 \%)$ patients had a further admission within one year due to other complications of injection drug use. Bacteraemia, S. aureus disease, septic shock, qSOFA/SIRS, inflammatory markers, metastatic infection and local abscess were not associated with clinical failure on univariable analyses. Duration of intravenous, inpatient or total antimicrobials was also not associated with outcome (Table S5, Fig. 1B).

\section{Discussion}

i-DVT in PWID was frequently associated with bacteraemia, groin abscesses and septic pulmonary emboli. Patients often received lengthy antimicrobial courses associated
B

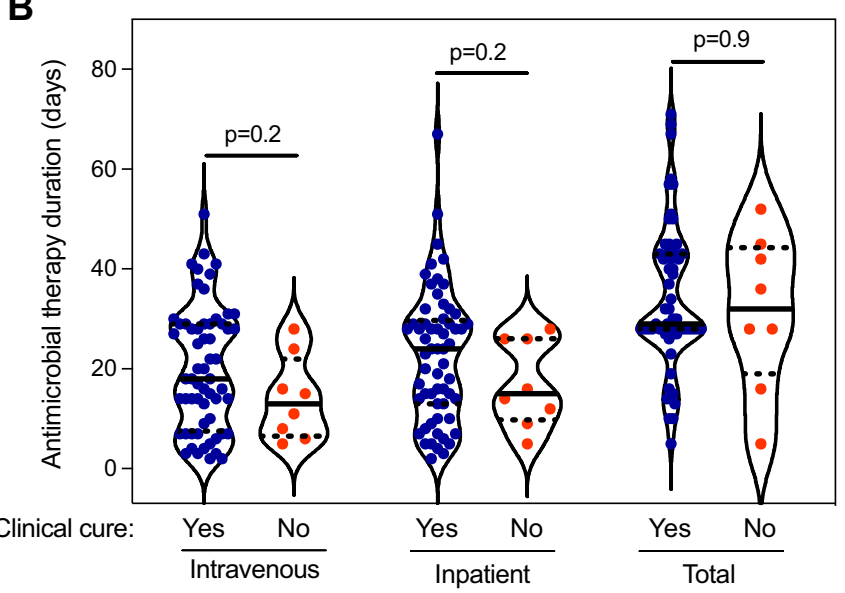

D

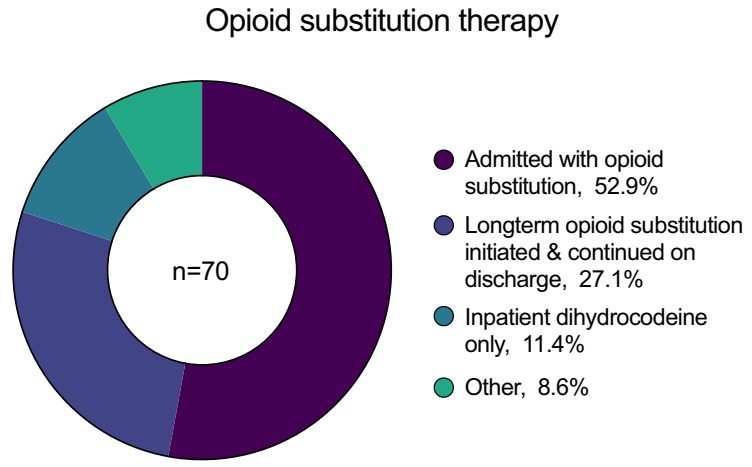

ney test. Solid line within violin plot shows median and dotted lines show first and third quartiles. C Initial spectrum of empiric antimicrobial therapy. Definitions of different spectra are presented in Supplementary Table 3. D Opioid substitution therapy

with extended hospitalisations. Despite the complex nature of infections, outcomes were surprisingly good, with clinical cure in $88.6 \%$ of patients. Moreover, cure occurred independent of highly variable durations of antimicrobial therapy. Patient characteristics and outcomes reported here are similar to other smaller studies (Table S6) $[1,5,6]$. In a North American cohort of PWID with invasive bacterial infections (predominantly IE and bone/joint infections), outcomes were similar for patients who completed a prolonged intravenous antimicrobial course and those who required partial oral therapy (and superior to those who did not complete intravenous therapy nor receive partial oral therapy) [7]. Combined with data supporting partial oral therapy for the treatment of IE and osteomyelitis (in studies excluding PWID [8, 9]), we contend there exists a rationale to devise pragmatic approaches to implement novel individualised treatment plans utilising oral antimicrobial therapy for i-DVT. A microbiologic diagnosis was frequently achieved (49/70 patients). S. aureus, streptococci and anaerobes were the most commonly identified pathogens whereas 
gram-negative pathogens were identified very infrequently (in 3/49 patients with a microbiological diagnosis). Considering the rarity of identifying gram-negative pathogens, we propose that routine use of broad spectrum/gram-negative therapy empirically is not required.

Although treatment failure of the index infection was uncommon, readmissions within one year with other complications of injection drug use occurred in almost half the cohort (45.7\%), justifying intensification of efforts to address causation. Fourteen patients $(20 \%)$ were discharged without addressing opioid substitution therapy. Active HCV was common (31.4\%) but only a minority were successfully engaged in treatment. There was minimal documentation regarding harm reduction measures. In Scotland, drugrelated acute admissions are increasing steadily with 10,509 admissions in 2017-18 (199 stays/100,000 population), representing 7,986 distinct patients of which $54 \%$ had not been admitted before[10].

The generalisability of these results is limited by the single-centre design. Our ability to identify predictors of treatment failure was limited by the low event rate. Patients re-admitted to hospitals outwith the geographic area of the study would not have been identified so the figures presented for clinical cure represent an upper bound (i.e. we may have missed cases of clinical failure); however local experience suggests this is unlikely to have been a frequent event.

This report addresses a deficit in the clinical and microbiological characterisation of i-DVT in PWID, providing a basis for reformed antimicrobial approaches. Despite frequent interactions and prolonged hospital stays, we also identify missed opportunities to address causal factors and more specifically to target the reservoir of active HCV in PWID. PWID presenting acutely with i-DVT represent a high-risk group in need of an integrated, holistic approach to management and efforts to facilitate healthcare engagement.

Supplementary Information The online version contains supplementary material available at https://doi.org/10.1007/s15010-021-01725-3.

Acknowledgements We gratefully acknowledge the assistance of Bill Juliff and Robyn Pascoe at NHS Lothian Analytical Services for performing the electronic patient record search to identify possible patients.

Author contributions HM, CDR, DTOS: Conceptualization, Methodology, Writing - Review \& Editing, Project administration. HM, CDR: Investigation, Formal analysis, Data Curation, Writing-Original Draft.

Funding H.M. and C.D.R. are supported by Edinburgh Clinical Academic Track (ECAT)/Wellcome Trust PhD Training Fellowship for Clinicians awards (H.M. 220406/Z/20/Z; C.D.R. 214178/Z/18/Z).
Availability of data and material Anonymised raw data available on reasonable request.

Code availability Not applicable.

\section{Declarations}

Conflicts of interest None.

Ethics approval This work was approved by the NHS Lothian Infection Service Quality Improvement Team.

Open Access This article is licensed under a Creative Commons Attribution 4.0 International License, which permits use, sharing, adaptation, distribution and reproduction in any medium or format, as long as you give appropriate credit to the original author(s) and the source, provide a link to the Creative Commons licence, and indicate if changes were made. The images or other third party material in this article are included in the article's Creative Commons licence, unless indicated otherwise in a credit line to the material. If material is not included in the article's Creative Commons licence and your intended use is not permitted by statutory regulation or exceeds the permitted use, you will need to obtain permission directly from the copyright holder. To view a copy of this licence, visit http://creativecommons.org/licenses/by/4.0/.

\section{References}

1. Mertz D, Khanlari B, Viktorin N, Battegay M, Fluckiger U. Less than 28 days of intravenous antibiotic treatment is sufficient for suppurative thrombophlebitis in injection drug users. Clin Infect Dis. 2008;46(5):741-4.

2. Heldman AW, Hartert TV, Ray SC, et al. Oral antibiotic treatment of right-sided staphylococcal endocarditis in injection drug users: prospective randomized comparison with parenteral therapy. Am J Med. 1996;101(1):68-76.

3. Rodger L, Glockler-Lauf SD, Shojaei E, et al. Clinical characteristics and factors associated with mortality in first-episode infective endocarditis among persons who inject drugs. JAMA Netw Open. 2018;1(7):e185220-e.

4. Freeman TC, Horsewell S, Patir A, et al. Graphia: A platform for the graph-based visualisation and analysis of complex data. bioRxiv 2020: 2020.09.02.279349.

5. Hakeem MBD. Septic deep venous thrombophlebitis and distant emboli in injecting drug users - treatment experience and outcome. J R Coll Phys Edinb. 2007;37(4):293-9.

6. Fäh F, Zimmerli W, Jordi M, Schoenenberger RA. Septic deep venous thrombosis in intravenous drug users. Swiss Med Wkly. 2002;132(27-28):386-92.

7. Marks LR, Liang SY, Muthulingam D, et al. Evaluation of partial oral antibiotic treatment for persons who inject drugs and are hospitalized with invasive infections. Clin Infect Dis. 2020;71(10):e650-6.

8. Li HK, Rombach I, Zambellas R, et al. Oral versus intravenous antibiotics for bone and joint infection. N Engl J Med. 2019;380(5):425-36.

9. Iversen K, Ihlemann N, Gill SU, et al. Partial oral versus intravenous antibiotic treatment of endocarditis. $\mathrm{N}$ Engl $\mathrm{J}$ Med. 2019;380(5):415-24.

10. NHS National Services Scotland, Information Services Division. Drug-Related Hospital Statistics Scotland 2017/18, 2019. 